\title{
Zur Gletscher- und Klimageschichte des alpinen Spätglazials
}

\author{
Dem Andenken an meine liebe Freundin Karin Fanghänel † gewidmet
}

\section{Einleitung}

Als die Alpengletscher nach der letzten großen eiszeitlichen Vorstoßphase durch die nun einsetzende Klimaverbesserung nach und nach zurückzuschmelzen begannen, wurde eine Periode eingeleitet, die wir als das alpine Spätglazial bezeichnen. Nach der Gliederung des Würm-Glazials von M. WELTEN (1981) stellt es innerhalb des "Spät-Würms», das den Zeitabschnitt von rund 25-13000 resp. 10000 Jahren BP umfasst (S. 10, Tabl. 1), gleichsam den «Endabschnitt der letzten Kaltphase und Vergletscherung» dar (S. 12). Der durch die spätglaziale Erwärmung verursachte Gletscherrückgang sowie die damit einhergehende Wiederbesiedlung durch die Vegetation verliefen jedoch nicht kontinuierlich, sondern wiesen mehrere Abschnitte auf. Wiederholte Klimarückschläge bewirkten, daß die Gletscherströme regenerierten, erneut vorstießen und dabei die sog. "Stadiale» bildeten. Klimaverbesserungen (sog. "Interstadiale») ließen das nunmehr in einzelne Talgletscher zerfallende Eisstromnetz weiter ins Alpeninnere zurückschmelzen. Dadurch konnten die in der WürmEiszeit verdrängten und zum Teil vernichteten Pflanzengesellschaften wieder einwandern und das nun eisfrei werdende Gelände sukzessive wiedererobern. Wann genau der Rückgang der Gletscher von den würmzeitlichen Stirnbereichen und damit definitionsgemäß der Beginn des Spätglazials einsetzte, ist im einzelnen jedoch noch weitgehend unklar und konnte bisher weder glazialmorphologisch noch pollenanalytisch an irgendeiner Lokalität erfaßt werden. Selbst über die Reichweiten und Endlagen des letzten bedeutenden, vor etwa 20000 Jahren erfolgten WürmVorstoßes sind die Auffassungen gerade durch neuere Untersuchungen wieder stark im Wandel begriffen. So hält z. B. M. welten $(1979$, S. 29) die damalige Gletscherausdehnung - entgegen der früheren Lehrmeinung - für wesentlich kleiner (etwa dem Bern- bzw. Zürich-Stadium entsprechend) als das mittlerweile ins Früh-Würm zurückverlegte Würm-Maximum. Als Zeitpunkt für den Beginn des Spätglazials gibt M. WELTEN (1979, S. 32) für das Aaretal rund 15000 Jahre vor heute an. G.PATZELT (1980, S.13) hingegen veranschlagt dafür in den Ostalpen rund 16-17000 Jahre BP, einen Zeitraum, der durch Datierungen im Gebiet des Trauntales (D. vAN HUSEN 1977) bestätigt scheint. Das Ende des Spätglazials bzw. der Übergang ins Postgla- zial ist wesentlich besser bekannt und wird allgemein und übereinstimmend mit 10200 oder mit rund 10000 Jahren BP (vgl. J. MANGERUD et al. 1974) angenommen. Nach den heutigen Erkenntnissen ist dieser markante Klimaumschwung gekennzeichnet durch den endgültigen Rückgang der Gletscher auf etwa neuzeitliche bzw. postglaziale Größenordnung und mit dem Anstieg der Waldgrenze auf ungefähr heutige Höhenlage (G. PATZELT 1973, 1977).

Eine möglichst genaue und lückenlose Geschichte des spätglazialen Gletscher- und Vegetationsgeschehens in den Alpen zu entwerfen, ist schon seit langer Zeit das erklärte Forschungsziel zahlreicher in- und ausländischer Untersuchungen. Die vor allem in den rund 15 letzten Jahren in vermehrtem Maße durchgeführten Arbeiten über das alpine Spätglazial konnten immer mehr die Annahme erhärten, daß der Gletscherrückgang in seinen wesentlichsten Grundzügen und Merkmalen in den Ost- wie auch in den Westalpen nach durchaus ähnlichem Muster verlaufen ist. Die in verschiedenen Gletschergebieten der Alpen festgestellten, gegliederten und zum Teil datierten Ereignisse lassen sich heute in ein immer dichter belegtes allgemeines Schema einordnen, dessen Gültigkeit stets aufs neue bestätigt oder allenfalls regional verfeinert werden kann.

Trotz der Vielzahl von Einzelergebnissen, wie sie vor allem aus Österreich und der Schweiz vorliegen, gibt es bei einer kritischen Bestandesaufnahme der Resultate noch viele offene Fragen, die unbedingt noch einer exakteren Abklärung bedürfen. Damit sind auch in Zukunft weitere und intensivere Untersuchungen über das spätglaziale Gletscher- und Klimageschehen notwendig. In den folgenden Ausführungen soll nun versucht werden, die Entwicklung, die Probleme und den Stand der Spätglazial-Forschung in den, wie es uns scheint, wichtigsten Schritten und Gesichtspunkten aufzuzeigen und zu veranschaulichen. Aus einleuchtenden Gründen können die folgenden Erörterungen lediglich als eine vereinfachte Zusammenfassung der derzeit bekannten Resultate gelten. Für ein genaueres Studium der hier jeweils nur kurz erwähnten Einzelsituationen sei deshalb auf die zitierte Literatur verwiesen.

Dr. Max Maisch

Geographisches Institut der Universität Zürich

Postfach, 8033 Zürich 

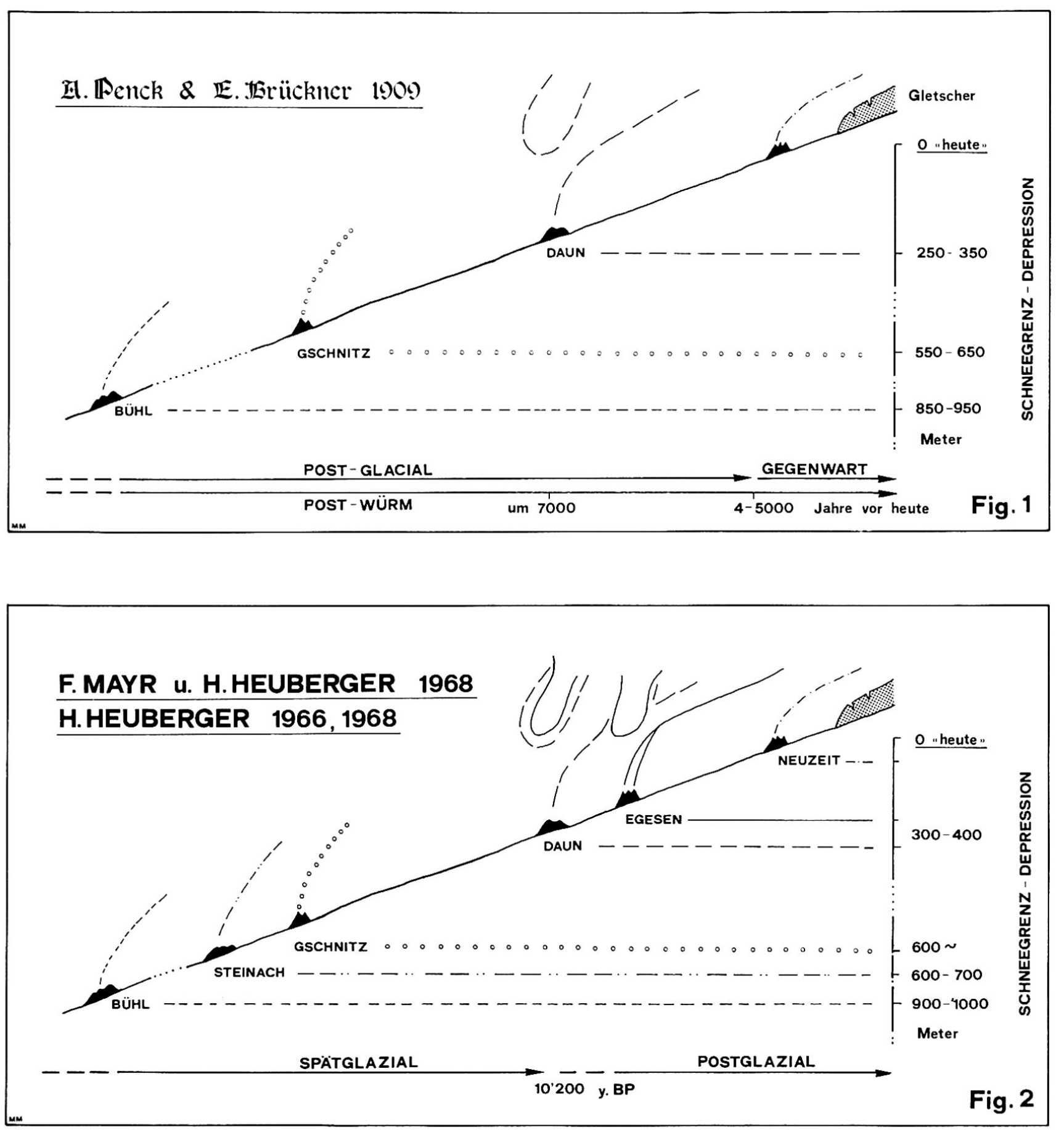

\section{Die Gliederung der Gletscherstadien}

\subsection{Entwicklung der Auffassungen}

Eine Gliederung der oft als "Rückzugsstadien» bezeichneten Wiedervorstöße wurde erstmals von A.PENCK und E.BRÜCKNER in ihrem berühmten dreibändigen Werk «Die Alpen im Eiszeitalter» (1909) nach Typlokalitäten im Raume des eiszeitlichen Inngletschers erarbeitet. Ihre klassische und seither allgemein bekannte Dreigliederung "Bühl-Gschnitz-Daun» (vgl. Fig. 1) repräsentiert dabei wesentliche Phasen des «postglacialen» Eisabbaues (tabellarische Übersichten der Typlokalitäten finden sich u.a. in H.HEUBERGER 1968, G.PATZELT 1972, M. MAISCH 1981 sowie F. RENNER 1982). Während zur Zeit des Bühl-Stadiums das inneralpine Eisstromnetz noch weitgehend intakt war, so zeigt die für das Gschnitz-Stadium maßgebende "Trinser-Moräne» bereits eine Situation an, bei der die Eismassen in mehrere selbständige Talgletscher zerfallen waren. Im Daun-Stadium existierten sodann in den hintersten Abschnitten der Seitentäler und in einzelnen Karen nur noch sog. "Lokalgletscher" (vgl. A. PENCK und E. BRÜCKNER 1909, S. 340, Fig. 60). Diese drei für den 

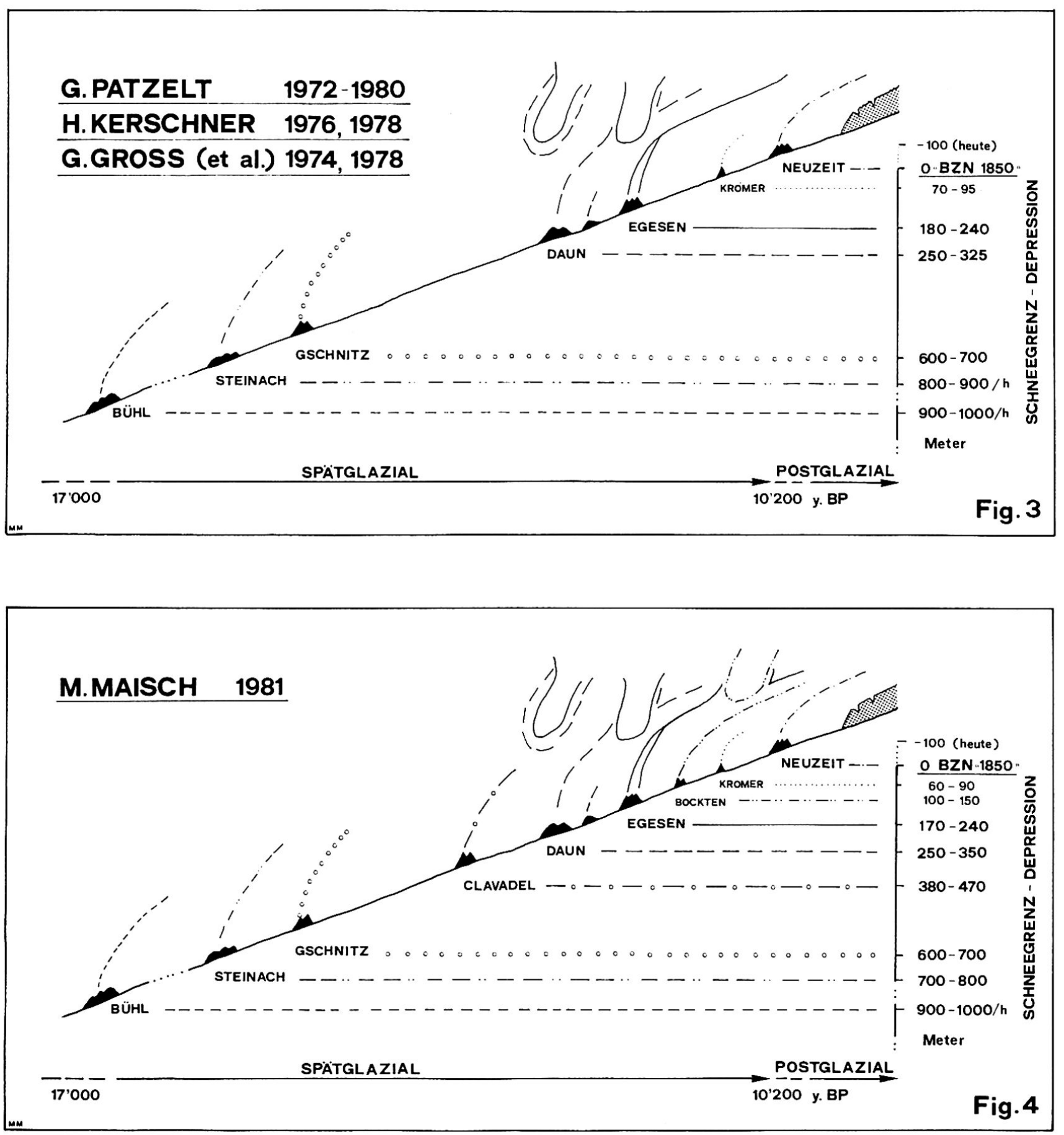

Gletscherrückgang als typisch erkannten Gletscherstadien waren jedoch nicht nur auf den Raum des Tiroler Inntales beschränkt, sondern sie konnten von PENCK und BRÜCKNER unter anderem auch in den "Helvetischen Gletschersystemen" der Rhone, Aare, Reuss, Linth und des Rheins lokalisiert werden (Bd.II, S.624ff.). Maßgebend für die Zuordnung zu einem der drei namengebenden Stadien waren neben der relativen Lage innerhalb der als würmzeitlich eingestuften «Jungendmoränen» vor allem Abschät- zungen der "Schneegrenzdepressionen» (Sg-Depr.) gegenüber der "heutigen» Höhenlage der Schneegrenze (vgl. Fig.1). Die Depressionswerte der einzelnen Stadien gruppierten sich dabei innerhalb charakteristischer Größenordnungen (Bd.III, S. 1164). Dahinter steht der auch heute noch gültige Grundgedanke, da $\beta$ Gletscherstände in vergleichbaren Klimaregionen (vgl. dazu J. SUTER 1982) mit gleichen oder zumindest ähnlichen Schneegrenzdepressionen letztlich auch zur gleichen Zeit stattgefunden haben. 
DaS PENCK und BRÜCKNERSChe Stadialsystem bildete in den nun folgenden glazialmorphologischen wie auch geologischen Arbeiten lange Zeit gültigen Ausgangspunkt für die Gliederung und Parallelisierung der Gletscherstände. Doch obwohl E. BRÜCKNER (Bd. II, S. 636) mit allem Nachdruck betonte, «...daß es nur drei Stadien giebt, nicht mehr", wurde ihr erstes Stadial-Schema mehrmals in Frage gestellt, umgedeutet, ergänzt, schließlich aber auch wieder bestätigt (vgl. dazu G. PATZELT 1972, 1975). Die von F.MAYR und H.HEUBERGER (1968) durchgeführte Neuuntersuchung (vgl. Fig. 2) erbrachte an den Typlokalitäten des Bühl- und des Gschnitz-Stadiums eine vollumfängliche Bestätigung bzw. Wiedereinführung dieser namengebenden Vorstoßphasen. Zudem konnten sie das von R.v. KLEBELSBERG (1950) entdeckte und von w.v.SENARCLENS-GRANCY (1958) näher beschriebene Steinach-Stadium gutheißen und auch dessen regionale Gültigkeit nachweisen (H. HEUBERGER 1966). In bezug auf das Daun-Stadium gelang sodann H. HEUBERGER (1966), vor allem durch morphologische Unterscheidungskriterien, eine "scharfe Zweiteilung» (S. 105) in eine ältere und eine jüngere Serie. Für die ältere Moränengruppe, die in der Regel durch solifluidal überformte und eher undeutlich und lückenhaft erhaltene Formen charakterisiert werden kann, behielt H. HEUBERGER den alten, damit in seiner Bedeutung aber etwas eingeschränkten Ausdruck "Daun-Stadium» bei. Die jüngere Serie mit zumeist frischen, blockreichen und deutlich erhaltenen Moränenwällen, die an geeigneten Stellen diskordant über Daun-Moränen liegen, wird von H. HEUBERGER mit dem bereits von H.KINZL $(1929,1932)$ eingeführten Begriff "Egesen-Stadium» bezeichnet. Ein schönes Beispiel für die Formunterschiede zwischen Daunund Egesen-Moränen ist in Abb. 1 dargestellt. Das gewöhnlich stets markanter als das Daun ausgeprägte Egesen-Stadium konnte seither praktisch in allen neueren gletschergeschichtlichen Arbeiten unterschieden werden.

In den hauptsächlich in den 70er Jahren an der Universität Innsbruck entstandenen Arbeiten wurde die von MAYR und HEUBERGER fixierte Abfolge "BühlSteinach-Gschnitz-Daun-Egesen» im Prinzip ohne Einschränkungen übernommen und auch in einem erweiterten räumlichen Rahmen bestätigt (G. PATZELT 1972, 1975, 1976, 1978, 1980, H. KERSCHNER 1976, 1978). Als ein Fortschritt bei der Gliederung und Parallelisierung spätglazialer Gletscherstände aufgrund von Schneegrenzdepressionen darf dabei sicher die systematische Erarbeitung der sog. "2-1-Flächenteilungsmethode» gelten. Die Anwendung dieses Verfahrens als Korrelationsmittel bietet dabei erstmals eine einheitliche, vergleichbare und letztlich auch glaziologisch abgestützte Grundlage (G. PATZELT 1973, 1975, H.KERSCHNER 1976, 1978, G.GROSS et al. 1978). Als Bezugswert für die Berechnung von Schneegrenzdepressionen wurde nun nicht mehr auf die als eher unzuverlässige bezeichnete "heutige» Schneegrenze zurückgegriffen (vgl. dazu G. GRoss et al. 1978, S. 240), sondern das "Bezugsniveau» (BZN) des neuzeitlichen Hochstandes von 1850 definiert (H. KERSCHNER 1976). Das Bezugsniveau liegt im Mittel zwischen 100-150 m tiefer als die "heutige» Schneegrenze. Die mit dieser neuen Methode errechneten Schneegrenz-Wertbereiche sind für die entsprechenden Stände in Fig. 3 dargestellt.

Die statistische Auswertung der Schneegrenzwerte ergab, da $\beta$ sich die Beziehungen zwischen dem Bezugsniveau 1850 und der Höhenlage der Gleichgewichtslinie der einzelnen Gletscherstände in Form von linearen Regressionsgleichungen darstellen lassen (H.KERSCHNER 1976, 1978, G.GROSS et al. 1978, M. MAISCH 1981). Das Steigungsmaß der Geraden enthält dabei indirekte Aussagen über die räumliche Verteilung der Depressionswerte in den verschiedenen Klimagebieten der Alpen. Die Depressionswerte für das Egesen-Maximalstadium wären dabei nach G. GRoss et al. (1978, S. 224) gesamtalpin nicht konstant gewesen, sondern hätten im feucht-maritimen Bereich der Nordabdachung im Mittel 300-320 m, im trocken-kontinentalen Zentralalpenbereich hingegen nur $180-220 \mathrm{~m}$ betragen. Diese räumliche Veränderlichkeit der Schneegrenzdepressionen wurde bereits von E. BRÜCKNER (1909, Bd. II, S. 636) für das DaunStadium (noch im alten Sinne) festgestellt und mit der individuellen Lage und der unterschiedlichen Niederschlagsverteilung erklärt. H.KERSCHNER (im Druck) interpretiert diesen Sachverhalt unter dem Gesichtspunkt einer gegenüber heute deutlich verschiedenen Niederschlagsverteilung. Der Niederschlag in $2000 \mathrm{~m}$ Höhe hätte dabei im Zentralraum zur Zeit des Egesen-Stadiums nur ungefähr $70 \%$ der heutigen Menge betragen, während er an der Nordflanke ungefähr gleich der heutigen gewesen sein soll. Diese Werte konnten von M. KUHN (im Druck) auf anderem Wege weitgehend bestätigt werden.

Durch systematische Schneegrenzberechnungen an Gletschern der Silvretta-Gruppe wurde von G.GRoss (1974) als letzter deutlich außerhalb der neuzeitlichen Ausdehnung liegender Vorstoß innerhalb der EgesenSerie neu der "Kromer-Stand" mit Sg-Depr. von 70-95 m eingeführt (vgl. G. GRoss et al. 1978, S. 245). Stände ähnlicher Merkmale und 2:1-Schneegrenzwerte konnten seither von F. BEELER (1977, «Morteratsch-Stand»?, Berninagruppe), R. носнноLD (1978, zit. in: R. SCHMID 1981, "Taubenkar-Stand», Trauntal), R. FRAEDRICH (1979, «Kartell-Schwankung», Ferwallgruppe), J.suter (1981, «Beverin-Stand», Err-JulierGruppe), M.MAISCH (1981, Mittelbünden), F. RENNER (1982, Zentralschweiz) und w. BIRCHER (1982 in Vorb., Saastal) nachgewiesen werden. Damit ist die weitere Verbreitung eines allgemeinen Gletschervorstoßes in der Größenordnung des namengebenden, weil erst erwähnten Kromer-Standes belegt.

Die eigenen, im Raume Mittelbünden zwischen Landwasser- und Albulatal durchgeführten Untersuchungen (M. MAISCH 1981) ergaben einerseits wieder- 
um eine vollumfängliche Bestätigung des MAYR und HEUBERGERschen Spätglazial-Systems. Für die äquivalenten Stände des Steinach-Gschnitz-Daun- und Egesen-Stadiums (inkl. Kromer) konnten nicht nur die wichtigsten morphologischen Merkmalskriterien, sondern, mit nur geringen Abweichungen der Variationsbreiten und Mittelwerte, auch die von G. GRoss et al. (1978) angegebenen Schneegrenzdepressionen bestätigt werden (Fig.4). Innerhalb dieser offenbar generell gültig scheinenden Stadialabfolge gelang es aber anderseits, zwei weitere Gletschervorstoßperioden nachzuweisen. Die als "Clavadel-Stadium" bezeichneten Moränensituationen im namengebenden vorderen Sertigtal (Davos) und in rund einem Dutzend weiterer Täler stellen dabei eine räumlich und mit 2:1-Schneegrenzdepressionen zwischen rund 380-470 m/BZN 1850 wohl auch klimatisch und damit auch zeitlich zwischen Gschnitz- und Daun-Stadium einzuordnende Vorstoßphase der Gletscher dar. Die mehrgliedrige Moränenstaffel bei Clavadel eignet sich dabei sehr gut als Typlokalität, konnte doch hier vom selben Gletscher sowohl das Gschnitz- wie auch das Daun-Stadium nachgewiesen werden. Damit scheint eine Verwechslung oder Fehlinterpretation der stadialen Abfolge höchst unwahrscheinlich.

Ebenfalls in mehreren Tälern Mittelbündens zeichnet sich innerhalb des Egesen-Maximalstadiums (rund 170-240 m Sg-Depr.) und vor dem Kromer-Stand (rund 60-90 m Sg-Depr.) mit rund 100-150 m Schneegrenzdepression ein weiterer deutlicher und mehrgliedriger Gletscherstand ab. Nach einer modellhaften Moränensituation (vgl. M. MAISCH 1981, S.45, Abb. 5) wurde er mit dem Lokalnamen "Bocktentälli-Stand" bezeichnet. Die in diesem Seitenkar lehrbuchmäßig ausgebildete Schüttungsdiskordanz zwischen den geschlossen erhaltenen Bocktentälli- und den EgesenMaximal-Moränen des Haupttalgletschers (Chüealptal) zeigt sehr schön, daß es sich bei diesem Ereignis um einen deutlichen $W$ iedervorsto $B$ gehandelt haben muß, dem eine eindeutige, allerdings unbestimmt lange Zeit des Abschmelzens vorangegangen war.

Damit lassen sich die in topographisch günstigen, d. h. meist flachen Tälern (vgl. M. MAISCH und w. HäBERLI 1982) bis über ein Dutzend zählenden Egesen-Stände morphologisch und damit gletschergeschichtlich in drei Hauptabschnitte gliedern: Egesen-Maximal-, Bocktentälli- und Kromer-Stand. Diese Abfolge entspricht generell der bereits von G.PATZELT (1977, S. 249, Tab. 1) vermuteten Dreigliederung des EgesenStadiums, nur wird an jener Stelle nicht konkret auf die einzelnen Vorstoßphasen eingegangen.

Da diese zwei neu definierten Vorstoßphasen des Clavadel-Stadiums und des Bocktentälli-Standes gleich an mehreren Stellen nachgewiesen werden konnten, ist es durchaus denkbar, daß es sich dabei nicht nur um regionale Ereignisse handelt, sondern $\mathrm{da} ß$ sie auch über diesen räumlichen Rahmen hinaus gültig sind. Hinweise über Stände ähnlicher Größenordnung liegen seither von F. RENNER (1982) aus dem
Gotthardgebiet, w. BIRCHER (1982 in Vorb.) aus dem Saastal (Wallis) und von R. vUAGNEUX (in Vorb., mdl. Mittlg.) aus dem Flüelatal (Davos) vor. Für eine endgültige Bestätigung vor allem des Clavadel-Stadiums, das, nach der Mächtigkeit, dem Erhaltungszustand und der Vielgliedrigkeit der Moränen zu schließen, eine überaus markante und kaum übersehbare Vorstoßphase darstellt, müssen in Zukunft weitere Alpentäler kartiert und nach einheitlichen Korrelationskriterien (2:1-Verfahren) miteinander verglichen werden. So wird es sich dann erweisen, ob die in der älteren Literatur noch gemäß dem PENCK und BRÜCKNERSchen Schema generell dem Gschnitzoder dem Daun-Stadium zugeordneten Moränenwälle mit den heute vorhandenen und als typisch erachteten Schneegrenzwerten übereinstimmen oder dann allenfalls umgedeutet werden müssen.

\subsection{Allgemeines über die Gliederung der Stadien}

Der vereinfachte, mit den eigenen Untersuchungsergebnissen verglichene historische Überblick hat gezeigt, daß die Kenntnisse über den Ablauf des spätglazialen Gletschergeschehens seit dem einfachen PENCK und BRÜCKNERSchen Modell immer differenzierter geworden sind und heute von einem wesentlich vielfältigeren Verhalten der Gletscher und damit des Klimas zeugen. Bemerkenswert ist jedoch die Tatsache, daß die von A. PENCK und E. BRÜCKNER (1909) aufgestellte Stadialabfolge "Bühl-GschnitzDaun» heute noch, allerdings erweitert und verfeinert, gültig ist und mit den ursprünglichen Typlokalitäten übereinstimmt (vgl. Fig. 1-4).

$\mathrm{Ob}$ mit den in der Zwischenzeit neu eingeführten Gletscherstadien nun sämtliche bedeutenden spätglazialen Gletschervorstöße erkannt worden sind, bleibt hingegen offen. So führt z.B. G. PATZELT in seiner Publikation von 1975 (S. 327, Fig. 53) zwischen Bühlund Steinach-Stadium erstmals ein "Pinzgau-Stadium» auf, ohne allerdings auf die Typlokalität oder deren besondere Situation einzugehen. Da dieser neue Gletschervorsto $B$ in den folgenden Arbeiten jedoch an keiner weiteren Stelle mehr Erwähnung findet (G. PATZELT und s. BORTENSCHLAGER 1978, g. PATZELT 1980), darf angenommen werden, $\mathrm{da} \beta$ es sich dabei um eine inzwischen aufgegebene oder dann nur streng lokale Gletscherschwankung handelt.

Daraus kann nun aber nicht abgeleitet werden, daß es zwischen Bühl- und Steinach-Stadium keine weiteren allgemeinen Gletschervorstöße mehr gegeben hat. Im Gegenteil: Wenn man einmal mit der PENCK und BRÜCKNERschen Auffassung übereinstimmt, daß das Bühl-Stadium im Bereich des vereinigten Linth- bzw. Rheingletschers bei «Hurden» (Bd. II, S. 528) resp. "... unter allen Umständen oberhalb des Bodensees...» (Bd. II, S. 435) anzusetzen ist, so schalten sich nach der Zusammenstellung von R. HANTKE (1978, 
S. 377) bis zum «Filisurer-Stadium», das gut mit dem Steinach-Stadium übereinzustimmen scheint (M. MAISCH 1981, S. 117), mit den Stadien von "Weesen»" (= "Feldkirch»), "Ragnatsch», "Sargans», "Chur» und "Tiefencastel" (vgl. R. HANTKE 1978, S.252, 373) mindestens fünf weitere markantere Vorstoßphasen ein. Selbst wenn die Bühl-äquivalenten Endlagen möglicherweise nicht genau stimmen und wohl auch nie ganz eindeutig festlegbar sind, zeigt diese Abfolge, $\mathrm{da} \beta$ es im Bereich zwischen Bühl- und Steinachähnlichen Moränensituationen noch weitere, bisher in der Diskussion über die Gliederung des Spätglazials kaum berücksichtigte Gletscher- und damit auch Klimaschwankungen gegeben hat. Auch in anderen Einzugsgebieten der Schweizer Alpen hat R. HANTKE zwischen den «Inneren Jungendmoränen» und den innersten spätglazialen Ständen weit mehr Stadien aufgelistet, als bisher im Bereich der namengebenden Ostalpen definiert wurden. Dort scheinen offenbar zumindest zwischen Bühl und Steinach einige Moränenserien nicht ausgebildet, nicht erhalten oder noch nicht erfaßt worden zu sein. Ob sich allerdings diese Stände, die ja meist noch von großen, kompliziert zusammengesetzten und damit äußerst schwierig rekonstruierbaren Gletschern gebildet wurden, nach irgendwelchen Kriterien je sicher korrelieren lassen, scheint aber eher unwahrscheinlich. Neuere und einigermaßen zuverlässige Angaben über die 2:1Schneegrenzdepressionen liegen bisher lediglich bis zum Gschnitz- (G. PATZELt 1975, G. GRoss et al. 1978, R. FRAEDRICH 1979, M. MAISCH 1981), mit zunehmender Unschärfe noch bis zum Steinach-Stadium vor (G. patzelt 1980, M. Maisch 1981). Für die Bezeichnung der noch älteren Stände kommen daher nach wie vor nur lokale oder allenfalls regionale Namen in Frage (vgl. R. HANTKE 1978, 1980).

Im Hinblick auf die klimageschichtliche Interpretation der stadialen Gletschergeschichte, z. B. beim Zeichnen einer spätglazialen Klimakurve, sei noch folgender Problemkreis angeschnitten. Anhand von Moränenrelikten lassen sich im Gelände lediglich Vorstoßphasen (evtl. längere Halte) erfassen, die man zudem noch modellhaft, im Trend der sukzessiven Erwärmung, als räumlich und zeitlich beinahe regelmäßig hintereinander gestaffelte Ereignisse interpretiert. Klimadepressionen, die das Ausmaß früherer Rückschläge übertrafen und bei denen die Gletscher eigene stadiale Moränen überfahren und zerstört haben - was zumindest theoretisch möglich erscheint -, bleiben damit glazialmorphologisch weitgehend unerkannt. Auch liegen, was für die Ableitung der damaligen $\mathrm{K}$ limaverhältnisse interessant wäre, über die $\mathrm{Ab}$ schmelzdistanzen und Mindestausdehnungen der Gletscher während der Interstadial-Zeiten (z. B. zwischen Daun und Egesen) nur wenige konkrete Anhaltspunkte vor. Damit können leider nur vage Aussagen über die darauf folgenden Vorstoßdistanzen und damit auch über die Heftigkeit und Intensität der Klimarückschläge gemacht werden.

\section{3. Über die Zeitstellung der Stadien}

Leider ist es bis heute nicht gelungen, eine der für die heutige Stadial-Gliederung maßgebenden Typlokalitäten direkt und gültig zu datieren. Bei der zeitlichen Einordnung der Stände ist man folglich, ähnlich wie bei der Schneegrenz-Methode, auf Analogie-Schlüsse angewiesen. Zudem liegen bei den bisher vorgenommenen Datierungsversuchen, mit nur wenigen Ausnahmen, lediglich "Mindestalter» vor. Diese Daten können strenggenommen mehrere Jahrhunderte (-tausende?) vom effektiven Zeitpunkt der Moränenbildung und des Eisfreiwerdens entfernt liegen und tragen dann, verglichen mit bereits vorhandenen Zeitmarken, nichts mehr zur Abstützung der Spätglazial-Chronologie bei. Im Gegensatz zu den postglazialen bzw. neuzeitlichen Gletscher- und Klimaschwankungen, die mittlerweile durch eine enorme Datendichte abgestützt werden konnten (vgl. M. GAMPER und J. SUTER 1982, HP. HOLZHAUSER 1982, beide dieses Heft), sind die Hinweise über die Altersstellung spätglazialer Moränen als eher spärlich zu bezeichnen und zusätzlich oft noch mit vielen Fragezeichen behaftet.

A. PENCK (1909, Bd. III, S. 1168ff.) nimmt an, daß das Bühl-Stadium auf rund 16-24000 Jahre vor heute zurückzudatieren sei. Für die Dauer der gesamten "Post-Würm-Zeit", die in unserem heutigen Sinne sowohl das Spät- wie auch das Postglazial umfaßt, veranschlagt er als Mittelwert rund 20000 Jahre (Fig. 1). Das Daun-Stadium, das A. PENCK «... für älter als die Pfahlbauten im allgemeinen» hält und für dessen Ende er den Zeitraum von spätestens 4-5000 Jahren angibt (Bd.I, S.382), setzt er aus archäologischen Überlegungen vor das Pfahlbauerneolithikum, also vor rund 7000 Jahren an (Bd. III, S. 1169).

H. HEUBERger $(1966,1968)$ ordnet das GschnitzStadium, in Anlehnung an Resultate aus dem Nordischen Vereisungsgebiet, der Jüngeren Dryas zu. Das Steinach-Stadium, das er anhand des Bodens von «Roppen» (Alleröd?) vom Gschnitz-Stadium trennt, stellt er in die Ältere Dryaszeit. Das Daun-Stadium fällt nach dieser Chronologie damit noch ins Postglazial, "wohl erst nach der Jüngeren Dryas", das Egesen-Stadium wird zwischen Jüngerer Dryas und postglazialer Wärmezeit angesetzt (Fig. 2). Gestützt wurde diese Zuordnung im wesentlichen durch die umfangreichen und maßgebenden Resultate von $\mathrm{H}$. ZOLLER et al. (1966).

Zahlreiche ${ }^{14} \mathrm{C}$-Datierungen von Moorbasisproben, wie sie seit Beginn der 70er Jahre von der Österreicher Schule durchgeführt und interpretiert wurden, ergaben für das Egesen- und damit auch für das DaunStadium erstmals den Nachweis vor-präborealen, d. h. spätglazialen Alters (G. PATZELT 1972). Egesen und Daun wurden vorerst gemeinsam der Jüngeren Dryas, das Gschnitz-Stadium der Älteren Dryas zugeordnet. Damit widerspiegelt die aus vegetationskundlichen und biostratigraphischen Untersuchungen schon 
längst bekannte Grenze Spät-/Postglazial gletschergeschichtlich sinnvoll gerade diejenige Periode, in der die Gletscher begonnen haben, sich endgültig und rasch auf neuzeitliche Größe zurückzuziehen (G. PATZELt 1972, S. 52). Weitere Mindestdatierungen aus österreichischen Gletschergebieten konnten seither die vermutete Gleichsetzung von Egesen und Jüngerer Dryaszeit weiter abstützen (vgl. G. PATZELt und s. BORTENSCHLAGER 1976, H. KERSCHNER 1978).

Aus der Schweiz liegen aus den neueren Arbeiten folgende Altershinweise für das Egesen bzw. für dessen Substadien vor:

\section{Berninapaßgebiet (F. BEELER 1977, 1981)}

Moor Moräne «Palü» (2070 m): $9635 \pm 160$ (UZ-46)

Moor «Palü» (1940 m): $9460 \pm 140$ (B-2635)

Beide Daten stellen Mindestalter für das der Stellung und Morphologie nach dem Egesen entsprechende «Cavaglia-Stadium» des Palügletschers (1977, S. 261) dar. Letzteres Datum zeigt, daß bereits um Mitte Präboreal, nach der vorangegangenen «Palü-Schwankung» (Schlaten?), der Gletscher nicht mehr viel größer war als bei den neuzeitlichen Hochständen.
Zentralschweiz (F. RENNER 1982 und schriftl. Mittlg.) Moor «Höhenbiel» (1970 m): $9730 \pm 120$ (UZ-119) Lage im Zungenbereich des Egesen-äquivalenten Standes «Ebnen» des Witenwasserengletschers. Die pollenanalytische Auswertung durch M. KÜTTEL (in Vorb.) ergab an der Basis eine Sedimentation bereits ausgangs der Jüngeren Dryas.

Moor «Torta» (2150 m): $\quad 9995 \pm 110$ (UZ-348)

Lage $1,3 \mathrm{~km}$ vor dem 1850er Hochstand. Das Datum stellt ein Mindestalter für den Egesen-Maximalstand, höchstwahrscheinlich auch für den Bocktentälli-äquivalenten Stand des Tortagletschers dar. Damit sind diese beiden Vorstoßphasen bereits am Ende der Jüngeren Dryas abgeschlossen.

Val de Nendaz, Wallis (M. KÜTTEL 1979 a.c. H. N. MÜLLER et al. 1982)

Moor «Tortin» (2039 m): Pollenanalyse

Lage des Moores innerhalb einer jüngeren Randlage (? Kromer) des Stadiums "Crouye Sina» (Egesen) des Tortingletschers. Das Basissediment reicht bis Ende Jüngere Dryas zurück und liefert ein Mindestalter für diesen internen Stand.

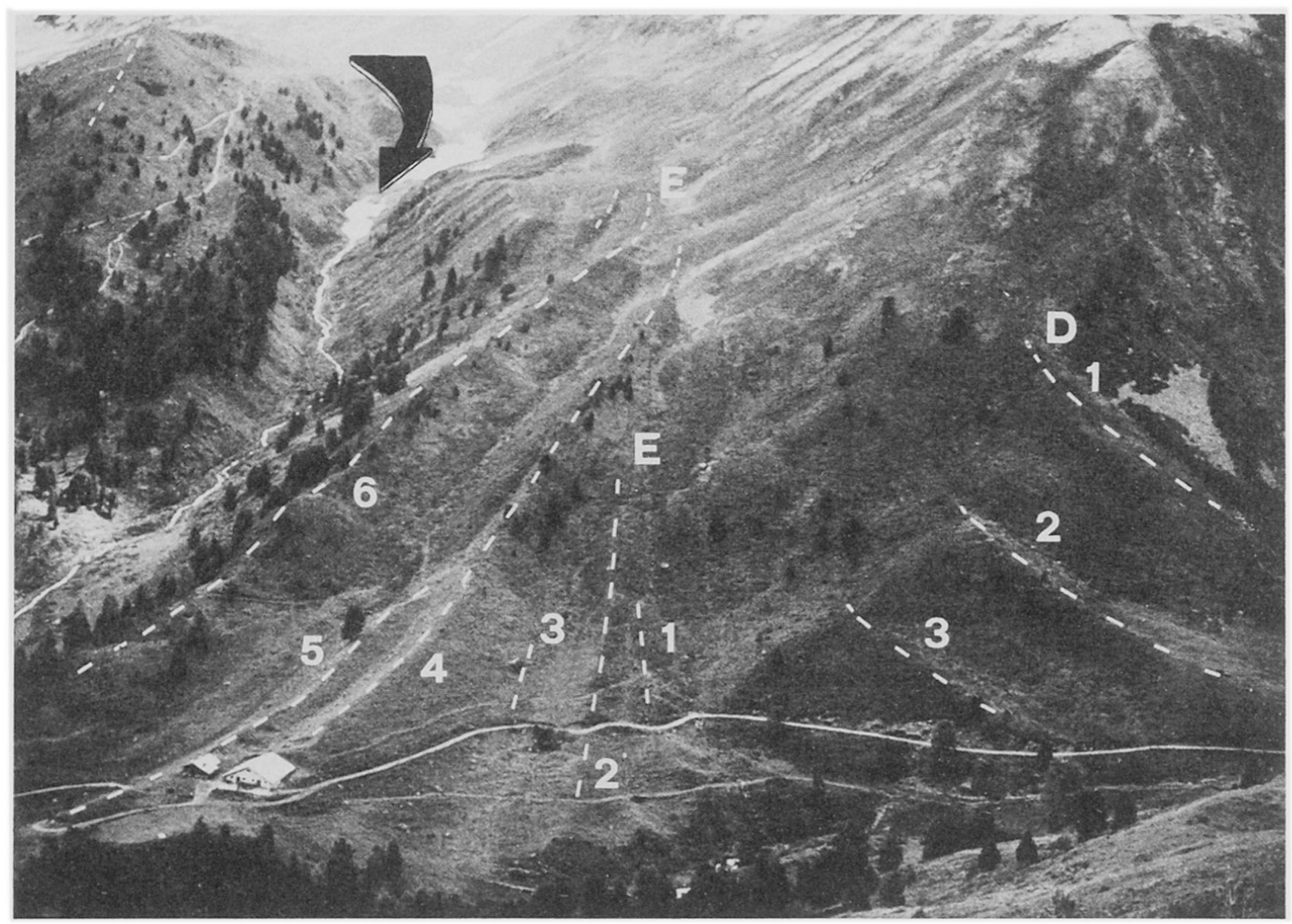

Abb.1 Spätglaziale Moränenwälle des Porchabellagletschers auf Alp digl Chant (Val Tuors, Bergün).

Deutlich lassen sich die solifluidal überprägten Wälle D 1-3 (Daun) und die mächtig und scharfgratig ausgebildeten Moränen $E$ 1-6 (Egesen) erkennen. 
Saastal, Wallis (w. BIRCHER 1982 und schriftl. Mittlg.) Moor «Wildi» (1800 m): $\quad 9760 \pm 175$ (UZ-141)

Pollenanalyse

Das Moor liegt ca. $1,5 \mathrm{~km}$ vom $1850 \mathrm{er}$ Hochstand entfernt. Das ${ }^{14} \mathrm{C}$-Datum liefert ein Mindestalter für den dem Egesen zugeordneten Stand "Saas Grund» des Feegletschers. Die von c. BURGa vorgenommene Pollenanalyse umfaßt zusätzlich sicher die Jüngere Dryas, evtl. sogar einen Erosionsrest eines allerödzeitlichen (?) Profilabschnittes. Im Profil zeichnet sich zudem das unmittelbare Überfahren der ehemaligen Felswanne durch den Gletscher während der Jüngeren Dryaszeit (evtl. Egesen-Maximalstand «Saas Grund» oder ein letzter dem Egesen angehörender Vorstoß) ab. Das Moor "Wildi» ist die bisher einzige Stelle im Alpenraum, wo ein Egesen-Vorstoß stratigraphisch direkt mit der Jüngeren Dryas verknüpft werden konnte.

Oberengadin (A. HEITZ et al. 1982a, b, B.GAMPER in Vorb.)

Moore «Maloya» (1815 m): 7.21

$10550 \pm 140$ (B-3087)

$9880 \pm 200(\mathrm{~B}-3086)$

$\mathrm{MB}=$ Moorbasis

7.3

MB $9830 \pm 120(\mathrm{~B}-3085)$

MB $9420 \pm 120 \begin{gathered}(\mathrm{B}-3408) \\ (\mathrm{B}-3407)\end{gathered}$

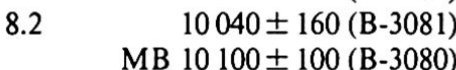

Die «Moor 7» bzw. «Moor 8» genannten Probeentnahmestellen liegen innerhalb des "Maloya-Stadiums» (Egesen) des Fornogletschers (vgl. B. GAMPER in Vorb.) und sind als Mindestalter zu interpretieren. Zur Überprüfung der parallel dazu untersuchten Pollenprofile wurden stratigraphisch dicht übereinander liegende ${ }^{14} \mathrm{C}$-Datenserien bestimmt. Reihe 7.21 zeigt dabei eine als "schwerwiegend" bezeichnete Inversion, Reihe 7.3 ist nach A. HEITZ et al. (1982b, Kap. 3.2) allgemein viel zu jung ausgefallen. Diese nur ausschnittsweise wiedergegebenen Datierungen zeigen eindrücklich, welche Vorsicht bei der Interpretation von einzelnen, nicht durch pollenanalytische Untersuchungen abgesicherten und isoliert stehenden ${ }^{14} \mathrm{C}$ Daten geboten sein muß. Mögliche Ursachen für diese ${ }^{14} \mathrm{C}$-Altersschwankungen sind in A. HEITZ et al. (1982b) nachzulesen.

Trotz diesen zuletzt gemachten Einschränkungen gibt es nun, unter Berücksichtigung der Zeitspanne zwischen Eisfreiwerden und dem Beginn der organischen Sedimentation, mehrere gute Hinweise, daß das Egesen-Stadium sicher vor dem Präboreal und damit in der Jüngeren Dryas stattgefunden hat, ja daß sogar die Hauptphase (Egesen-Maximalstand) mit gutem Grund an den Beginn der Jüngeren Dryaszeit zurückzuverlegen ist. Die einzigartige Situation im Moor "Wildi» (w. BIRCHER 1982) zeigt erstmals definitiv, daß das Egesen-Stadium tatsächlich in der Pollenzone III erfolgte und nicht etwa, wie das aufgrund der bisherigen Mindestalter eigentlich nicht ganz auszuschließen war, sogar noch älter einzustufen ist. Auch die zweite markante Egesen-Phase, der Bockentälli-Stand, scheint nun nach dem Befund im Moor «Torta» noch in die Jüngere Dryas zu fallen und bereits vor Beginn des Postglazials beendet gewesen zu sein. Falls die «jüngere Randlage» des "Crouye Sina-Stadiums» tatsächlich dem Kromer-Stand entspricht - leider fehlt dazu eine Schneegrenzberechnung - könnte auch dieser Vorstoß noch im Spätglazial stattgefunden haben. Dann wäre er sogar noch älter als der bisher dafür vermutete Abschnitt des frühen Präboreals (10200-9500 BP), das damit nach wie vor und im vermehrten $\mathrm{Maße}$ einen "gletscher- und klimageschichtlich unbefriedigend erfaßten Zeitraum» darstellt (G. Gross et al. 1978, S. 245). Die Problematik dieses Zeitraumes wird aus pollenanalytischer Sicht u. a. von M. KüTTEL $(1977,1979 b)$ diskutiert.

Die auf morphologischer Basis beruhende Unterteilung des Egesen-Stadiums in mehrere Vorstoßphasen scheint sich auch in vielen bisher vorliegenden Pollendiagrammen abzuzeichnen. Nach M. KÜTTEL (1982) lassen sich dabei im Zeitraum der Jüngeren Dryas anhand der Artemisia-Werte häufig 3-4 Gipfel nachweisen. Ob sich allerdings diese einzelnen Pollenschwankungen je mit bestimmten Gletscherspuren verknüpfen und mit den drei bisher unterschiedenen Egesen-Substadien in Verbindung bringen lassen, bleibt zu hoffen, scheint aber eher fraglich zu sein.

Für das Daun-Stadium gibt es bisher kein einziges gültiges ${ }^{14} \mathrm{C}$-Datum, das die vermutete und morphologisch begründbare Trennung von der Jüngeren Dryas und dem daran geknüpften Egesen-Stadium belegen könnte. Das einzige Datum, das bisher, wenn auch mit Vorbehalten, mit dem Daun-Stadium in Verbindung gebracht wurde (H. KERSCHNER 1976, G. GROSS et al. 1978), nämlich die auf $10700 \mathrm{BP}$ datierte Moorbasis am Maloyapaß (H. KLEIBER 1974), wird nach umfangreichen Nachbestimmungen von A. HEITZ et al. (1982b, Kap. 3.2) als «etwas zu alt» interpretiert. Für die Altersstellung Daun-äquivalenter Gletscherstände liegen aus der Schweiz nunmehr aber folgende Hinweise vor:

Albulapaß (м. мAISCH 1981)

Moor «Crap Alv» (2045 m): Pollenanalyse

Das Moor liegt innerhalb des Daun-äquivalenten «Palpuogna-Standes» des Albulagletschers. Die von c. BURGa durchgeführte Pollenanalyse reicht an der Basis bis ins Alleröd oder zumindest bis in dessen Endphase zurück (S. 146).

Bernardinopaßgebiet (c. BURG A 1980, 1981)

Moor «Moräne Marschol» 1985 m): Pollenanalyse

Die Sedimentation im moränengestauten Moor (Stand «Nufenen» des Hinterrheingletschers) setzt mit großer Wahrscheinlichkeit während der Jüngeren Dryas oder kurz vor Ende des Alleröds ein (1981, S. 245). Dieser Hinweis stellt ein Mindestalter für diesen, dem Daun zugeordneten Gletscherstand dar. 
Das alpine SPÄTGL AZIAL

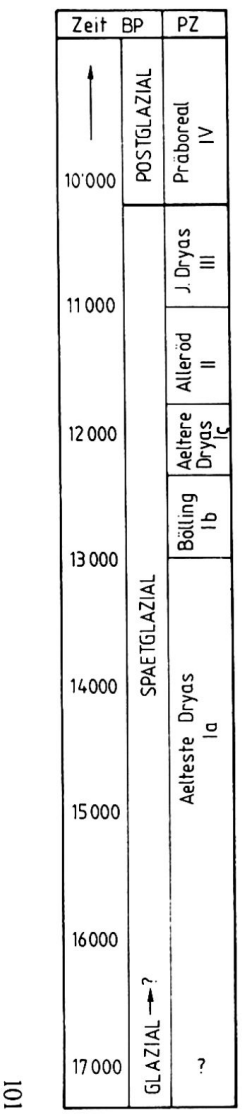

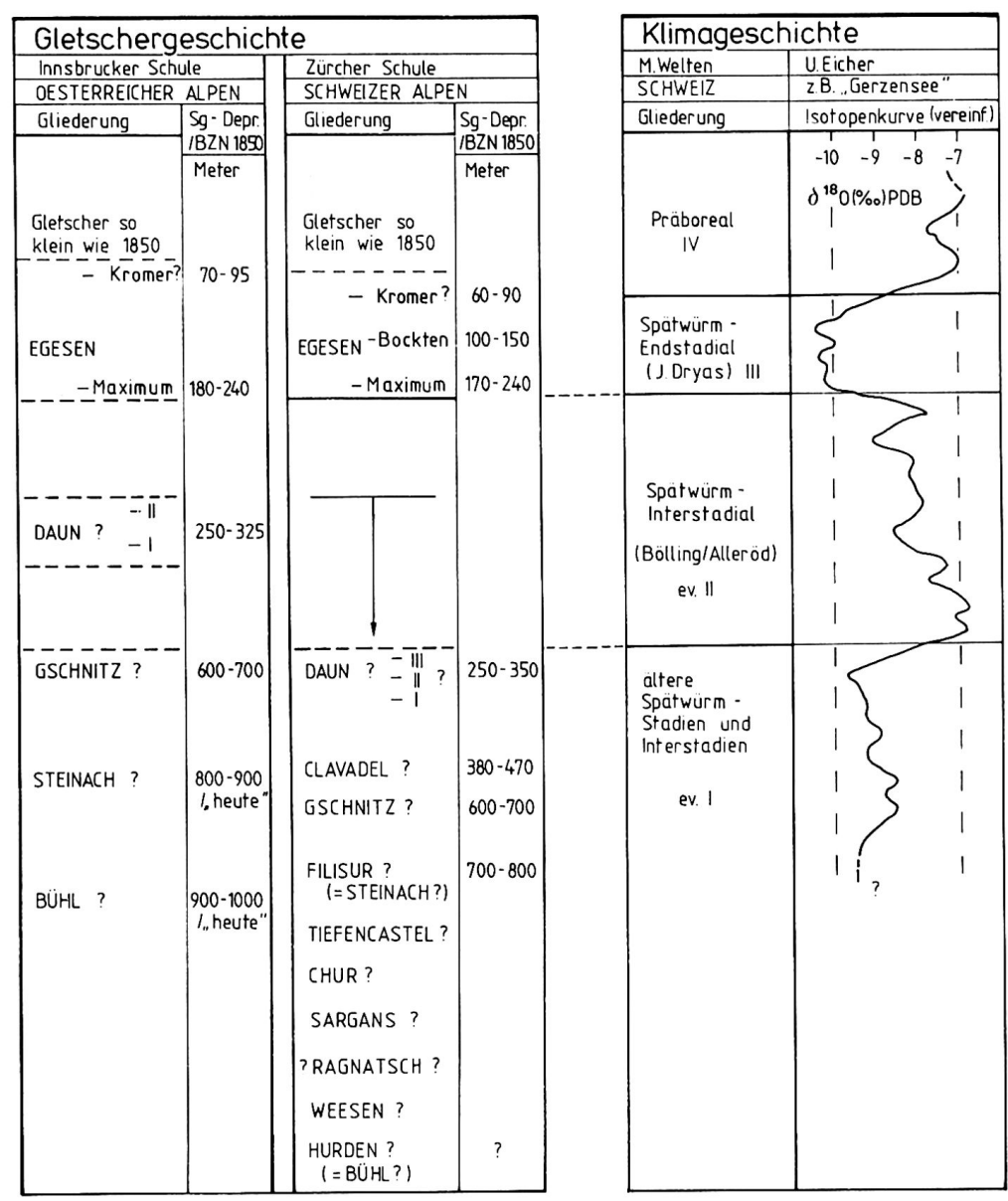

Vegetationsgeschichte

H.Zoller, H. Kleiber; C. Burga

Alpennordsei- Zentralalpen Alpensudsei\begin{tabular}{lll}
\hline te,Voralpen & VS, GR & te, Oberitalien \\
\hline
\end{tabular}

Pinus, Larix Pinus, Larix EMW, Betula

Pinus Cembra Pinus Cembra Pinus Cembra

\begin{tabular}{l|l|c} 
erstmals & Larix, Alnus \\
Ericaceae & glut/Inc
\end{tabular}

\begin{tabular}{l|l|l|}
\hline Parktundra: & Parktundra & Parktundra: \\
\hline
\end{tabular} Artemisia Artemisia Artemisia Ephedra,Chen Ephedra, Chen. Ephedra, Chen. Pinus, Betula Pinus, Betula Pinus, Betula \begin{tabular}{|l|l|}
\hline Wiederbewaldg dito bis & dito bis \\
\hline
\end{tabular}

mit Betula u. $1500 \mathrm{~m}$ mit $1600 \mathrm{~m}$

Pinus bis Betula, Pinus Larix, verm. über $1200 \mathrm{~m}$ etwas Larix Pinus Cembra

klimatisch bedingte Vegetationsveran derungen oft nur schwer nachweisbar

Parktundra: $\quad$ Steppentundra: Steppentundra Betula, Hippo- Betula, Larix Betula, Larix phae, Juniperus Hipp, Junip

\begin{tabular}{|l|l|l}
\hline Rohboden - & Rohboden - & Rohboden -
\end{tabular} \begin{tabular}{l|l|l|}
\hline Vogetation: & Vegetation & Vegetation \\
\hline
\end{tabular}

Artemisia Artemisia Artemisia

Ephedra Ephedra Ephedra

$\begin{array}{lll}\text { Ephedra } & \text { Ephedra } & \text { Ephedra } \\ \text { Chenopod. Chenopod. }\end{array}$

$\begin{array}{ll}\text { Chenopod. Chenopod. } & \begin{array}{l}\text { Chenopod. } \\ \text { lokal Junip }\end{array}\end{array}$

\begin{tabular}{l|l|l}
$?$ & $?$ & lokal Junip
\end{tabular}

Fig. 5

Max MAISCH

Geographica Helvetica, Nr. 2, 1982 
Oberengadin (A. HEITZ et al. 1982a, B. GAMPER in Vorb.) Moor «Mauntschas» (1818 m):

$12080 \pm 120$ BP (B-3606) Pollenanalyse

Das Moor liegt intramorän bezüglich des daunzeitlichen (?) Stadiums von "Samedan» des Berninagletschers (rund $300 \mathrm{~m} \mathrm{Sg-Depr.,} \mathrm{B.} \mathrm{GAMPER} \mathrm{mdl.} \mathrm{Mittlg.).}$ Die Basis des Moores enthält das Alleröd-Interstadial, aber auch präallerödzeitliche Horizonte (erste Pionierphase nach dem Rückzug des Berninagletschers). Wieweit diese aber noch in die Pollenzone I (Älteste Dryas) zurückreichen, ist pollenstratigraphisch nicht sicher bestimmbar. Das ${ }^{14} \mathrm{C}$-Basisdatum (Tongyttja) von $12080 \mathrm{BP}$ liegt stratigraphisch mitten im Abschnitt der Jüngeren Dryas und ist daher nach A. HEITZ et al. (Kap. 3.2.2) leider um 1000-2000 Jahre zu alt ausgefallen.

Mit diesen drei aus dem Bündnerland stammenden pollenstratigraphischen Hinweisen scheint es nun erstmals gerechtfertigt, das Daun-Stadium auch zeitlich vom Egesen zu trennen und vor dem AllerödInterstadial anzusetzen. Gegen eine den Befunden vorerst zwar logische und letztlich nicht ganz auszuschließende Zuordnung in die Ältere Dryas (Pollenzone Ic) oder sogar in eine innerallerödzeitliche Schwankung (? z.B. "Gerzensee-Schwankung», U. EICHER 1980) sprechen aber folgende Hinweise und Überlegungen (vgl. J.SUTER 1981, M. MAISCH 1981): In einer Vielzahl bisher im Alpenraum untersuchter Pollendiagramme fällt auf, daß sich der Zeitraum der Älteren Dryas nur sehr schwach und dann oft nur als Periode geringer Abkühlung abzeichnet (vgl. u.a. S. WEGMÜLLER 1977, R.E.SCHNEIDER 1978, F. KRAL 1979, U.EICHER 1979, 1980, U.EICHER et al. 1981, M. WeLten 1981, A. HEITZ et al. 1982a). Meist läßt sie sich dabei nur sehr schwer vom Bölling- und vom Alleröd-Interstadial abtrennen. Demgegenüber tritt das Bölling als markante Klimabesserung hervor und scheint sogar oft mit dem Alleröd eine einzige zusammenhängende Periode günstiger Klimawerte gewesen zu sein (U. EICHER 1979, M. WeLteN 1981). Nach den von U. EICHER aufgrund von SauerstoffIsotopen-Bestimmungen abgeleiteten Paläotemperaturkurven schnellen die späteiszeitlichen Temperaturen um 13000 BP steil hoch und erreichen dabei von Anfang an Werte, die denen des Postglazials sehr nahe kommen. Bis 11000 BP sinken sie dann mit Schwankungen kleineren Ausmaßes leicht ab, liegen zwischen 11000 und 10000 BP 2 bis $3^{\circ}$ tiefer, um zu Beginn des Postglazials wieder anzusteigen (vgl. M. WELTEN 1981, S. 13). Aus diesen Befunden gliedert M. Welten (1981, S. 13, Tab. 2) die spätwürmzeitliche Klimaentwicklung neu in drei Hauptabschnitte: die älteren Spätwürm-Stadien und Interstadien (vor 13000 BP), zwischen 13000 und 11000 BP eine einheitliche warme Phase (Bölling/Alleröd-Interstadial) und zwischen 11000 und $10000 \mathrm{BP}$ das "Spätwürm-Endstadial» der Jüngeren Dryas. Diese Dreigliederung wird gestützt bzw. bestätigt durch die Untersuchungen von
U. EICHER (1980), U. EICHER et al. (1981), U. EICHER und U. SIEGENTHALER (1982) sowie Von M. KÜTTEL (1982).

Aus diesem Ablauf des Klimageschehens schließt bereits U. EICHER (1979, S. 194), daß das Einordnen bestimmter klassischer Rückzugsstadien in die Ältere Dryas mit einer gewissen Vorsicht erfolgen sollte. Alle diese Hinweise legen es nun nahe, das Daun-Stadium und damit auch alle älteren Gletschervorstöße sogar noch vor dem Bölling, also in der Ältesten Dryas, anzusetzen. Falls sich die Klimadepression der Älteren Dryaszeit überhaupt auf das Verhalten der Gletscher ausgewirkt hat, so dürften die Spuren dieses allfälligen Vorstoßes, wenn man vom Betrag des Temperaturrückganges direkt auf die Reichweite der Gletscher schließen darf, von den später einsetzenden Egesen-Vorstößen sehr wahrscheinlich überfahren worden sein. Das Daun-Stadium scheint damit, bis weitere Ergebnisse über mögliche Interstadial-Zeiten innerhalb der Ältesten Dryas vorliegen (vgl. M. KÜTTEL 1982), zeitlich und damit auch gletschergeschichtlich der Gschnitz/Clavadel-Serie näher zu liegen als dem Egesen-Stadium.

Für die älter als daunzeitlichen Gletscherstände gibt es gegenwärtig folgende Hinweise:

Oberengadin (J. SUTER 1981, A. HEITZ et al. 1982a)

Moor «Lej Marsch» (1820 m): $13850 \pm 490$ (UZ-301) $14320 \pm 80$ (B-3665) Pollenanalyse

Das Moor liegt innerhalb des «Cinuos-Chel-Stadiums" des Oberengadingletschers $(400-500 \mathrm{~m} \mathrm{Sg}$ Depr., Clavadel-äquivalent). Die ${ }^{14} \mathrm{C}$-Basisdaten stellen Mindestalter für dieses Stadium dar. Das Pollendiagramm umfasst nur gerade die Jüngere Dryas und in einer Seekreideschicht das ausgehende AllerödInterstadial. Nach A. HEITZ et al. (Kap. 3.2.2) sind die ${ }^{14} \mathrm{C}$-Datierungen im Vergleich dazu zu alt ausgefallen. Die Basisproben werden daher momentan nachdatiert (J. SUTER, mdl. Mittlg.). Da nun aber bereits das intern gelegene «Samedan-Stadium» (Daun?) als präallerödzeitlich und aus oben genannten Gründen auch präböllingzeitlich eingestuft werden muß, kommt als Ablagerungszeitraum für das "Cinuos-Chel-Stadium» mit gutem Grund nur noch die Älteste Dryas in Frage.

Damit muß nun aber der für das Gschnitz-Stadium bis anhin vermutete Zeitraum «wenige Jahrhunderte um oder knapp vor 13000 BP» (G. PATZELT 1978, S. 32) für wesentlich älter angenommen und die dafür maßgebende Situation des "Lanser-See-Profiles» in bezug auf die Gletschergeschichte möglicherweise neu interpretiert werden.

Auf eine gegenüber der Auffassung von G.PATZelt $(1975,1978,1980)$ etwas abweichende Zeitstellung des Gschnitz-Stadiums weist bereits D.v.HUSEN (1977) hin. Für den dem Gschnitz zugeordneten «GoisererStand» (Trauntal) belegt er ein Alter von rund 14000 BP. Wenn auch dieser Hinweis auf einer extrapolierten ${ }^{14} \mathrm{C}$-Datierung beruht und die Parallellisierung zum 
allgemeinen Spätglazial-Schema nicht mit 2:1Schneegrenzberechnungen abgesichert werden konnte, so muß das Gschnitz-Stadium auf alle Fälle um den Zeitraum vor 13000 Jahren zurückverlegt werden, den die Gletscher für die Vorstöße des Daunund des Clavadel-Stadiums bzw. für das Abschmelzen dazwischen benötigt haben. Im Vergleich zur Dauer des Egesen-Stadiums müssen dafür im Minimum 1000 Jahre, vermutlich aber eher mehr veranschlagt werden. Die Zeitstellung des Steinach- und des Bühl-
Stadiums liegt, abgesehen von einzelnen Hinweisen (vgl. D. v. HUSEN 1977), die den Zeitraum zwischen 16-17000 BP (Steinach?) bzw. um 17000 BP (Bühl?) nahelegen, noch weitgehend im unklaren.

Für eine weitergehende Abstützung der heutigen, zugegeben noch stark provisorischen SpätglazialChronologie sind damit in Zukunft noch weitere absolute Datierungen, wenn möglich in Zusammenhang mit pollenanalytischen Untersuchungen, anzustreben.

\section{Literatur}

BEELER F. (1977): Geomorphologische Untersuchungen am Spät- und Postglazial im Schweizerischen Nationalpark und im Berninapaßgebiet (Südrätische Alpen). Ergebnisse der wissensch. Untersuchungen im Schweiz. Nat. Park, Bd. XV, 131-276.

BEELER F. (1981): Das Spät- und Postglazial im Berninapaßgebiet. In: Geographica Helvetica, Heft Nr. 3, 101-108.

BIRCHER W. (1982) (in Vorb.): Klima- und Gletschergeschichte des Saastales: Glazialmorphologische und dendroklimatologische Untersuchungen. Diss. der Univ. Zürich.

BURGA C.A. (1980): Pollenanalytische Untersuchungen zur Vegetationsgeschichte des Schams und des San BernardinoPaßgebietes (Graubünden, Schweiz). Diss. Bot. 56, 165 S. + Anhang.

BURGA C.A. (1981): Glazialmorphologische Untersuchungen im Hinterrhein-Tal und am Bernardino-Paß. Vierteljahresschrift der Natf. Gesellschaft in Zürich, Heft 4, Jg. 126, 237-267.

EICHER U. (1979): Die ${ }^{18} \mathrm{O} /{ }^{16} \mathrm{O}$ - und ${ }^{13} \mathrm{C} /{ }^{12} \mathrm{C}$-Isotopenverhältnisse in spätglazialen Süßwasserkarbonaten und inr Zusammenhang mit den Ergebnissen der Pollenanalyse. Diss. Univ. Bern.

EICHER U. (1980): Pollen- und Sauerstoffisotopenanalysen an spätglazialen Profilen vom Gerzersee, Faulenseemoos und vom Regenmoos ob Boltigen. In: Mittlgen. der Natf. Ges. in Bern, N. F., Bd. 37, 65-80.

EICHER U., SIEGENTHALER U. and WEGMÜLLER S. (1981): Pollen and Oxygen Isotope Analyses on Late- and Post-Glacial Sediments of the Tourbière de Chirens (Dauphiné, France). In: Quarternary Research 15, 160-170.

EICHER U. und SIEGENTHALER U. (1982): Klimatische Informationen aus Sauerstoffisotopenverhältnissen in Seesedimenten. Phys. Geogr. Vol. 1, Zürich.

FRAEDRICH R. (1979): Spät- und postglaziale Gletscherschwankungen in der Ferwallgruppe (Tirol/Norarlberg). In: Düsseldorfer Geogr. Schriften, Heft 12, $161 \mathrm{~S}$.

FURRER G., HOLZHAUSER HP., MAISCH M. und SUTER J. (1982): Zur Geschichte unserer Gletscher im Spät- und Postglazial. In: Universität Zürich, Berichte zur Forschung. Zum 150-Jahr-Jub. der Univ. Zürich, Hersg. vom Wissensch. Inf.Dienst: 103-114.

GAMPER M. und SUTER J. (1982): Postglaziale Klimageschichte der Schweizer Alpen. In: Geographica Helvetica, Heft Nr. 2, 105-114.
GAMPER-SCHOLLENBERGER B., in Vorb.: Gletschergeschichte des Oberengadins: Untersuchungen von Gletscherschwankungen in der Bernina- und Languard-Gruppe. Diss. Univ. Zürich.

GROSS G. (1974): Schneegrenzberechnungen an den Gletschern der Silvrettagruppe. Hausarbeit aus Geographie, Univ. Innsbruck.

GROSS G., KERSCHNER H. und PATZELT G. (1978): Methodische Untersuchungen über die Schneegrenze in alpinen Gletschergebieten. In: Zeitschr. für Gletscherkunde und Glazialgeologie. Bd. XII, Heft 2, 1976: 223-251.

HANTKE R. (1978): Eiszeitalter 1. 468 S., Ott-Verlag Thun.

HANTKE R. (1980): Eiszeitalter 2. 703 S., Ott-Verlag Thun.

HANTKE R. (1983): Eiszeitalter 3. Ott-Verlag Thun.

HEITZ A., PUNCHAKUNNEL P. und ZOLLER H. (1982a): Vegetations-, Klima- und Gletschergeschichte des Oberengadins. Phys. Geogr. Vol. 1, Zürich.

HEITZ A., PUNCHAKUNNEL P. und ZOLLER H. (1982b): Zum Problem der ${ }^{14} \mathrm{C}$-Datierung im Veltlin und Oberengadin. Phys. Geogr., Vol. 1, Zürich.

HEUBERGER H. (1966): Gletschergeschichtliche Untersuchungen in den Zentralalpen zwischen Sellrain und Ötztal. In: Wissensch. Alpenvereinshefte, Heft 20,126 S., Innsbruck.

HEUBERGER H. (1968): Die Alpengletscher im Spät- und Postglazial. In: Eiszeitalter und Gegenwart, Bd. 19: 270-275, Innsbruck.

HOCHHOLD R. (1978): Die Gletscher der Dachsteingruppe. Geogr. Hausarbeit, Univ. Innsbruck.

HOLZHAUSER HP. (1982): Neuzeitliche Gletscherschwankungen. In: Geographica Helvetica, Heft Nr. 2, 115-126.

HUSEN VAN D. (1977): Zur Fazies und Stratigraphie der jungpleistozänen Ablagerungen im Trauntal. In: Jahrb. der Geol. Bundesanstalt, Bd. 120, Heft 1: $130 \mathrm{~S}$.

KERSCHNER H. (1976): Das Daun- und das Egesen-Stadium in ausgewählten Tälern der Zentralalpen von Nordtirol und Graubünden. Diss. Univ. Innsbruck.

KERSCHNER H. (1978): Untersuchungen zum Daun- und Egesen-Stadium in Nordtirol und Graubünden (methodische Überlegungen). In: Geogr. Jahresbericht aus Österreich, Bd. XXXVI (1975-1976): 26-49. Inst. für Geographie der Univ. Wien. 
KERSCHNER H. (im Druck): Outlines of the Climate during the Egesen-Advance (Younger Dryas, 11000-10000 BP) in the Central Alps of the Western Tyrol, Austria.

KINZL H. (1929): Beiträge zur Geschichte der Gletscherschwankungen in den Ostalpen. In: Zeitschrift für Gletscherkunde, Bd. XVII, Heft 1-3, 66-121.

KINZL H. (1932): Die größten nacheiszeitlichen Gletschervorstöße in den Schweizer Alpen und in der Mont Blanc-Gruppe. In: Zeitschrift für Gletscherkunde, Bd. XIX, Heft 4-5, 269-397.

KLEBELSBERG VAN R. (1950): Das Silltal bei Matrei. SchlernSchriften Bd. 84, 76-86.

KLEIBER H. (1974): Pollenanalytische Untersuchungen zum Eisrückzug und zur Vegetationsgeschichte im Oberengadin. In: Botan. Jb. für Syst. 94, 1, 1-53.

KRAL F. (1979): Spät- und postglaziale Waldgeschichte der Alpen aufgrund bisheriger Pollenanalysen. Veröff. des Inst. für Waldbau an der Univ. für Bodenkultur in Wien: $175 \mathrm{~S}$.

KÜTTEL M. (1977): Pollenanalytische und geochronologische Untersuchungen zur Piottino-Schwankung (Jüngere Dryas). In: Boreas, Vol. 6, 259-274, Oslo.

KÜTTEL M. (1979a): Pollenanalytische Untersuchungen zur Vegetationsgeschichte und zum Gletscherrückzug in den westlichen Schweizer Alpen. In: Ber. der Schweiz. Botan. Ges., Bd. 89, 1/2, 9-62.

KÜTTEL M. (1979b): Kritische Anmerkungen zu Nachweisen präborealer Klimaschwankungen. In: Peterm. Geogr. Mittlgen, $3 / 79$.

KÜTTEL M. (1979c): Räumliche und zeitliche Korrelation der “moraines intermédiaires» mit besonderer Berücksichtigung der Moränen der Alpage de Tortin (Nendaz, VS). In: Bull. de la Murithienne 96, 71-83.

KÜTTEL M. (1982): Veränderungen in der spätglazialen Umwelt von Luzern. Phys. Geogr., Vol. 1, Zürich.

KUHN M., (im Druck): Die Reaktion der Schneegrenze auf Klimaschwankungen.

MAISCH M. (1981): Glazialmorphologische und gletschergeschichtliche Untersuchungen im Gebiet zwischen Landwasserund Albulatal (Kt. Graubünden, Schweiz). Phys. Geogr., Vol. 3 , 215 S.

MAISCH M. und HÄBERLI W. (1982): Interpretation geometrischer Parameter von Spätglazialgletschern im Gebiet Mittelbünden, Schweizer Alpen. Phys. Geogr., Vol. 1, Zürich.

MANGERUD J., ANDERSEN S.T., BERGLUND B.E. and DONNER J.J. (1974): Quaternary stratigraphy of Norden, a proposal for terminology and classification. In: Boreas, Vol. 3 , 109-126, Oslo.

MAYR F. und HEUBERGER H. (1968): Types areas of Late Glacial and Post-Glacial Deposits in Tyrol, Eastern Alps. Proc. Congr. Quatern. In: Res. VII, Vol. 14, 143-165, Boulder, Colorado.

MÜLLER H.N., KERSCHNER H. und KÜTTEL M. (1982): Gletscher- und vegetationsgeschichtliche Untersuchungen im Val de Nendaz (Wallis) - Ein Beitrag zur alpinen Spätglazialchronologie. In: Zeitschr. für Gl.kunde und Glazialgeologie, Bd. 16. Heft 1, 1980, 61-84.

PATZELT G. (1972): Die spätglazialen Stadien und postglazialen Schwankungen von Ostalpengletschern. In: Ber. der dt. Botan. Ges., Bd. 85, Heft 1-4, 47-57.

PATZELT G. (1973): Die postglazialen Gletscher- und Klimaschwankungen in der Venedigergruppe (Hohe Tauern, Ostalpen). In: Zeitschr. für Geomorphologie, N. F. Suppl. 16, 25-72.
PATZELT G. (1975): Unterinntal-Zillertal-Pinzgau-Kitzbühel. Spät- und postglaziale Landschaftsentwicklung. In: Tirol, ein geographischer Exkursionsführer. Innsbr. Geogr. Studien, 2.

PATZELT G. (1977): Der zeitliche Ablauf und das Ausmaß postglazialer Klimaschwankungen in den Alpen. In: B. FRENZEL: Dendrochronologie und postglaziale Klimaschwankungen in Europa. Erdwissensch. Forschung, Bd. XIII, 248-259, Franz Steiner Verlag $\mathrm{GmbH}$, Wiesbaden.

PATZELT G. (1978): Das Gschnitz-Stadium bei Trins im Gschnitztal. In: Innsbrucker Raum und Ötztal. Führer zur TirolExkursion anl. der DEUQUA: 31-32.

PATZELT G. (1980): Neue Ergebnisse der Spät- und Postglazialforschung in Tirol. Jahresbericht 1976/77 Österr. Gesellschaft, Zweig Innsbruck: 11-18.

PATZELT G. und BORTENSCHLAGER S. (1976): Zur Chronologie des Spät- und Postglazials im Ötztal und Inntal (Ostalpen, Tirol). In: B. FRENZEL (Hrsg.): Führer zur Exkursionstagung des IGCP-Projektes 73/1/24 "Quaternary Glaciations in the Northern Hemisphere», Stuttgart: 120-135.

PATZELT G. und BORTENSCHLAGER S. (1978): Spät- und nacheiszeitliche Gletscher- und Vegetationsentwicklung im inneren Ötztal. In: Innsbrucker Raum und Ötztal. Führer zur Tirol-Exkursion anl. der DEUQUA: 13-25.

PENCK A. und BRÜCKNER E. (1909): Die Alpen in Eiszeitalter. 3 Bände, 1199 S., Leipzig.

RENNER F. (1982): Beiträge zur Gletschergeschichte des Gotthardgebietes und dendroklimatologische Untersuchungen an fossilen Hölzern. Diss. der Univ. Zürich.

SCHMIDT R. (1981): Grundzüge der spät- und postglazialen Vegetations- und Klimageschichte des Salzkammergutes (Österreich) aufgrund palynologischer Untersuchungen von See- und Moorprofilen. Mittlgen. der Komm. für Quartärforschung der Österr. Akademie der Wissenschaften, Bd. 3, 96 S.

SCHNEIDER R. (1978): Pollenanalytische Untersuchungen zur Kenntnis der spät- und postglazialen Vegetationsgeschichte am Südrand der Alpen zwischen Turin und Varese (Italien). In: Botan. Jahrb. Syst., Bd. 100, 1: 26-109, Stuttgart.

SUTER J. (1981): Gletschergeschichte des Oberengadins: Untersuchungen von Gletscherschwankungen in der Err-JulierGruppe. Phys. Geographie, Vol. 2, Zürich.

SUTER J. (1982): Zur klimatischen Interpretation der Gletschergeschichte. Phys. Geographie, Vol. 1, Zürich.

WEGMÜLLER S. (1977): Pollenanalytische Untersuchungen zur spät- und postglazialen Vegetationsgeschichte der französischen Alpen (Dauphiné). Verlag Paul Haupt, Bern.

WELTEN M. (1979): Eis, Wasser und Mensch haben das Aaretal verändert. Ergebnisse von 50 Jahren Pollenanalyse in Bern. In: Mittlgen. der Natf. Ges. in Bern, N. F., Bd. 36, 17-40.

WELTEN M. (1981): Gletscher und Vegetation im Lauf der letzten hunderttausend Jahre. Vorläufige Mitteilung. In: Gletscher und Klima. Jahrbuch der Schweiz. Nat.-Gesellschaft, wissenschaftl. Teil: 5-18, 1978, Birkhäuser Verlag, Basel/Boston/Stuttgart.

ZOLLER H. (1960): Pollenanalytische Untersuchungen zur Vegetationsgeschichte der insubrischen Schweiz. Denkschr. Schweiz. Natf. Gesellschaft, 83, 156 S.

ZOLLER H., SCHINDLER C. und RÖTHLISBERGER H. (1966): Postglaziale Gletscherstände und Klimaschwankungen im Gotthardmassiv und im Vorderrheingebiet. In: Verhandl. der Natf. Gesellschaft Basel, Bd. 77, Nr. 2, 97-164. 